\title{
MiR-106b exhibits an anti-angiogenic function by inhibiting STAT3 expression in endothelial cells
}

\author{
Ailifeire Maimaiti, Aikebaier Maimaiti, Yining Yang and Yitong Ma*
}

\begin{abstract}
Background: Recent discoveries of the atherosclerosis-related miRNAs shed new light on the treatment of cardiovascular diseases. Of note, miR-106b 25 cluster and miR-17 $~ 92$ cluster are paralogs. Up till now, plenty of researches have shown the role of miR-17 92 cluster in tumor and atherosclerosis, but miR-106b 25 cluster has stayed mysterious in atherosclerosis field. This study was designed to investigate how miR-106b functions in the atherosclerosis-related angiogenesis and to explore the functioning processes of miR-106b, so as to seek out a new target for the treatment of atherosclerosis.
\end{abstract}

Methods: Up and down regulation of miR-106b expression was achieved through transfection in HUVECs so as to investigate the function of miR-106b. Next we predicted the target genes of miR-106b and detected them using qRT-PCR and Western blot technique. At last, luciferase assay was conducted to verify the direct target gene of miR-106b. Data are expressed as mean \pm SEM. Two treatment groups were compared by Mann-Whitney $U$ test or student's $t$-test. Results were considered statistically significant when $P<0.05$.

Results: The results showed miR-106b up-regulation groups formed less tubes than control groups while the downregulation groups showed the opposite. Meanwhile, no obvious effect on apoptosis was observed in endothelial cells. Next we predicted the target genes of miR-106b and finally settled down to MAPK14 (Mitogen-Activated Protein Kinase), STAT3 (Signal Transducers and Activators of Transcription 3), JAK1 (Janus Kinase 1) and VEGFA(Vascular Endothelial Growth Factor A) as candidate target genes. Our results revealed over-expressed miR-106b represses STAT3 expression, while miR-106b inhibition resulted in STAT3 up-regulation. Ultimately, luciferase assay confirmed STAT3 mRNA is the direct target of miR-106b.

Conclusions: Our research demonstrated that miR-106b modulate angiogenesis in endothelial cells through affecting expression of STAT3, which occurs by direct target action. Therefore, we affirmed that miR-106b exerts an anti-angiogenic effect in endothelial cells via STAT3-involved signaling pathway, via directly targeting STAT3.

Keywords: Angiogenesis, Atherosclerosis, Endothelial cells, Micrornas

\section{Background}

Vascular generation also known as neovascularization refers to vasculogenesis, angiogegnesis and arteriogenesis [1, 2]. In this study, we focused mainly on the angiogenesis process Angiogenesis is the process of generating new capillary blood vessels. Physiologically, angiogenesis could be found in wound healing, infections, female reproductive system and also embryogenesis [2, 3]. Unregulated angiogenesis

\footnotetext{
* Correspondence: myt-xj@163.com

The Heart Center, the First Affiliated Hospital of Xinjiang Medical University, No.137 Liyushan South Road, Urumqi, Xinjiang, China
}

may result in different pathologies such as atherosclerosis, diabetic retinopathy, rheumatoid arthritis and tumor etc [2]. Many atherosclerotic lesions are vascularized by a network of capillaries that arise from the adventitial vasa vasorum. These capillaries may be important regulators of plaque instability. Reflecting their inflammatory microenvironment, the capillaries are immature endothelial tubes with disorganized branching, fragile and prone to rupture. The accumulation of erythrocytes after intraplaque hemorrhage which contains phospholipids and free cholesterol may promote stable plaque to transform into unstable lesion, 
increasing the probability of cardiovascular events [4]. Studies have shown that the proliferation rates of endothelial cells in plaque capillaries range from undetectable to $43 \%$, which indicates these vessels are found in various stages of development $[5,6]$, revealing the key role of endothelial cells in the whole process. Other studies showed that longterm treatment with recombinant murine endostatin or TNP-470 significantly reduced the further growth of atherosclerosis without affecting cholesterol levels [6], which strongly support that intimal capillaries contribute to the progression of atherosclerosis.

Recent discoveries of the atherosclerosis-related miRNAs provoked some thoughts about the diagnosis and treatment of cardiovascular diseases [7]. MiRNAs are a large class of evolutionarily conserved, small, noncoding RNAs, typically 22 nucleotides in length, which primarily function post-transcriptionally by interacting with the 3'untranslated region (UTR) of specific target mRNAs in a sequence-specific manner. MiRNAs have emerged as central regulators of many cardiogenic processes [8]. In the whole history of miRNAs studying, miR-17 92 cluster was extremely famous for its angiogenesis-related features, no matter in tumors or in atherosclerotic diseases. As its paralog, miR-106b 25 cluster's role in atherosclerosis still remain to be elucidated. Accordingly, our study mainly focused on how miR-106b act on its target in the angiogenesis processes in endothelial cells.

\section{Methods}

\section{Cell culture}

Human umbilical vein endothelial cells (HUVECs) were isolated from donated umbilical cords and cultivated in ECM medium (Sciencell,Cat.No. 1001) supplemented with ECGs (Sciencell, Cat. No. 1052) plus $5 \%$ FBS (Cat. No. 0025), and used until passage seven. 293 T cells were obtained from Guangzhou RiboBio.Co.Ltd and cultured under the recommended conditions.

\section{Transfection}

For transfection, HUVECs were cultured to $70 \%$ confluence and transfected with 30nM miR-106b mimic (Ambion, Cat. No.4464066) or miR-106 inhibitor (Ambion, Cat. No.AM17000) oligonucleotides using Lipofectin (Invitrogen, Cat. No.18292-011) according to the manufacturer's instructions. For miR-106b mimic transfection, there were three different treatment groups prepared, which were: mimic group (HUVECs transfected with miR106b mimic plus Lipofectin), mock group (HUVECs mixed with only Lipofectin) and negative-control group [HUVECs transfected with miRNA mimic negative control (Ambion, Cat. No.4464058) plus Lipofectin]; similarly, for transfection of miR-106b inhibitor, there were inhibitor group (HUVECs transfected with miR-106b inhibitor plus
Lipofectin), mock group (HUVECs mixed with only Lipofectin) and negative-control group [HUVECs transfected with miRNA inhibitor negative control (Ambion, Cat. No.4464076) plus Lipofectin]. For transfection of $293 \mathrm{~T}$ cells with STAT3 plasmids, miR-106b mimic was transfected with Lipofectamine 2000 (Invitrogen, Cat. No.11668027) to the plasmid/Lipofectamine ${ }^{\mathrm{TM}} 2000$ transfection mix with the final concentrations of 50nM.

\section{Tube formation assay}

Forty eight hours after transfection, HUVECs 200ul ( $\mathrm{C}=$ $2 \times 10^{5} / \mathrm{ml}$ ) from different treatment groups were cultured respectively in a 48-well plate (Corning) coated with $150 \mu$ l Matrigel Basement Membrane Matrix (BD Biosciences) in each well. The number of formed tubes was quantified after $24 \mathrm{~h}$ by counting the cumulative tube number in five random microscopic fields, the average number of each group was calculated and normalized to the average number of negative-control group.

\section{Flow cytometry analysis}

For fixation, HUVECs after transfection were detached with trypsin, fixed in $4 \%$ formaldehyde for $10 \mathrm{~min}$ and stored in $80 \%$ ethanol. For permeabilization, cells were incubated in $20 \mu \mathrm{g} / \mathrm{ml}$ proteinase $\mathrm{K}$ at room temperature for $5 \mathrm{~min}$ and then treated with $\mathrm{TdT}$ Labeling Reaction Mixture (MERCK, Cat. No.QIA39$1 \mathrm{EACN})$ at $37{ }^{\circ} \mathrm{C}$ for $1-1.5 \mathrm{~h}$ in the dark. Resuspend cells in $1 \times$ TBS and analyze cells on a FACS Canto II device (BD).

\section{RNA isolation and RT-PCR}

Total RNA of HUVECs was isolated using Qiazol (Qiagen, Cat. No.79306) according to the manufacturer's protocol. Subsequently, $1 \mu \mathrm{g}$ of RNA from each sample was reverse transcribed into cDNA and subjected to conventional PCR. To assess the transfection efficiency in HUVECs transfected with miR-106b mimic or inhibitor, we isolated total RNA using Qiazol 24 h after transfection. RT-PCR was performed using the TaqMan MicroRNA Reverse Transcription Kit (Applied Biosystems, Cat. No.4366596) and primers which are specific for reverse transcription of hsa-miR-106 and RNU44 (one cycle: $30 \mathrm{~min}$ at $16{ }^{\circ} \mathrm{C}$, $30 \mathrm{~min}$ at $42^{\circ} \mathrm{C}, 5 \mathrm{~min}$ at $85^{\circ} \mathrm{C}$ ). Real-time PCR was performed using the Taqman MicroRNA Assay (Applied Biosystems, Cat. No.4427975) and Taqman Universal PCR Master Mix II (Applied Biosystems, Cat. No.4440040) with RNU44 as loading control. (One cycle: $10 \mathrm{~min}$ at $95{ }^{\circ} \mathrm{C}$, 40 cycles: $15 \mathrm{~s}$ at $95^{\circ} \mathrm{C}, 60 \mathrm{~s}$ at $\left.60^{\circ} \mathrm{C}\right)$.

To assess the predicted target genes (MAPK14, STAT3, $V E G F A$ and $J A K 1)$ relative expression, we isolated total RNA as stated above and eliminated DNA with DNase I, Amp Grade (Invitrogen, Cat. No.18068-015). RT-PCR was performed using the High-Capacity cDNA Reverse 

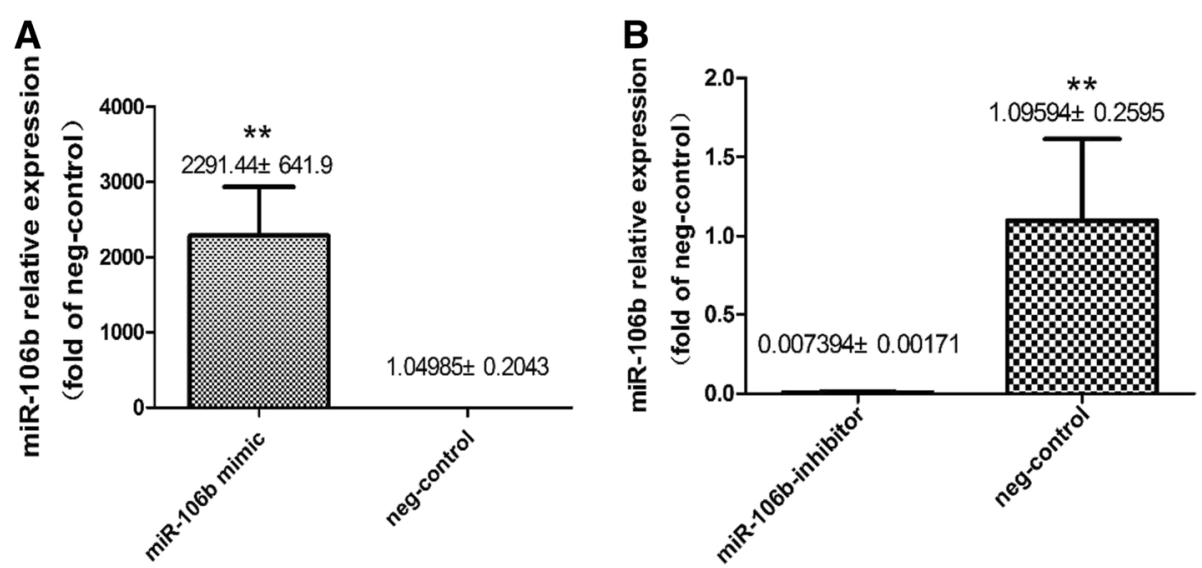

Fig. 1 a, b MiR-106b relative expression in different groups after transfection. Quantitative real-time PCR shows a strong increase in miR-106b in miR-106b mimic transfection group and a dramatic decline in miR-106b levels in miR-106b inhibitor transfection group, which explicitly guaranteed the transfection efficiency. MiR-106b levels were normalized to RNU44, Data are presented as means \pm SEM. **P $<0.05(n=4)$

Transcription Kits (Applied Biosystems, Cat. No. 4368814) (one cycle: $10 \mathrm{~min}$ at $25^{\circ} \mathrm{C}, 120 \mathrm{~min}$ at $37^{\circ} \mathrm{C}$, $5 \mathrm{~min}$ at $\left.85{ }^{\circ} \mathrm{C}\right)$. Real-time PCR was performed using the Power SYBR Green PCR Master Mix (Applied Biosystems, Cat. No.4367659) and primers specific for amplification of MAPK14, STAT3, VEGFA and JAK1 with GAPDH (Glyceraldehyde-3-phosphate dehydrogenase) as a reference gene. (One cycle: $10 \mathrm{~min}$ at $95{ }^{\circ} \mathrm{C}, 40 \mathrm{cy}-$ cles: $15 \mathrm{~s}$ at $95^{\circ} \mathrm{C}, 60 \mathrm{~s}$ at $\left.60{ }^{\circ} \mathrm{C}\right)$. The primers used were as follows: $M A P K 14$ : forward: $5^{\prime}$-GAGCGTTACCAGA ACCTGTCTC-3', reverse: 5'-AGTAACCGCAGTTCTC TGTAGGT-3'; STAT3: forward: 5'-CTTTGAGACCGAGGTGTATCACC-3', reverse: 5'-GGTCAGCATGT TGTACCACAGG-3'; VEGFA: forward: 5' -TTGCCTTG CTGCTCTACCTCCA-3', reverse: 5' -GATGGCAGTAG CTGCGCTGATA-3'; JAK1: forward: 5'-GAGACAGGT CTCCCACAAACAC-3', reverse: 5'-GTGGTAAGGAC ATCGCTTTTCCG-3';GAPDH: forward: 5'-GGCCTT CCGTGTTCCTACC-3', reverse: 5' -CGGCATGTCAGA TCCACAAC-3'.

\section{Western blot analysis}

For western blot analysis of STAT3 expression, cultured cells were lysed in RIPA (Radio-Immunoprecipitation Assay) buffer on ice and protein concentration was quantified using the Bradford method. Equal amounts of protein were loaded, subjected to SDS-PAGE (Sodium dodecyl sulfate polyacrylamide gel electrophoresis) and blotted on PVDF (Polyvinylidene Fluoride) transfer membranes. Blots were incubated over night with antibodies directed against human STAT3 or GAPDH as a loading control. Proteins were probed with primary antibodies and then with HRP (horseradish peroxidase)conjugated secondary antibodies. The enhanced chemiluminescence signal was detected using a densitometry program (ChemiDoc XRS, BioRad). To quantify the protein signal, we subtracted background and normalized the value to GAPDH with ImageLab 4.0.

\section{Luciferase reporter assay}

293 T cells (Guangzhou RioBobio Co Ltd, China) $\left(1.5 \times 10^{4}\right.$ cells/well) were seeded in 96-well plates. The predicted binding sequences on the 3'UTR of STAT3 were synthetically produced by PCR and transferred into a luciferase reporter vector. $100 \mathrm{ng}$ of STAT3 3' -UTR luciferase reporter plasmid (Guangzhou RioBobio Co Ltd, China) and either $50 \mathrm{nM}$ miR-106b mimic oligonucleotide or a non-targeting miRNA mimic control (Negative-Control) (Guangzhou RioBobio Co Ltd, China) were co-transfected. All transfections were performed using the Lipofectamine ${ }^{\mathrm{TM}} 2000$ in OPTI-MEM I Reduced Serum Medium (100 $\mu \mathrm{l} /$ well) for $24 \mathrm{~h}$. The luciferase activities from each well were measured using the Dual Glo Luciferase Assay System (Promega, USA) according to the manufacturer's instruction.

\section{Statistical analysis}

Data are expressed as mean \pm SEM. Two treatment groups were compared by Mann-Whitney $U$ test or student's $t$-test (GraphPad Prism 5.). Results were considered statistically significant when $P<0.05$.

\section{Results}

Modulation of miR-106b Expression in Cultured HUVECs

To explore the specific role of miR-106b in angiogenesis, we up or down regulated the expression of miR-106b in endothelial cells by using specific miR-106b mimic or inhibitor in transfection, respectively. Mimics and inhibitors with irrelevant nucleotide sequences served as negative controls. We detected transfection efficiency by quantitative real-time PCR which showed a strong increase in miR-106b at $24 \mathrm{~h}$ after the mimic transfection, whereas transfection with inhibitor resulted in 
A

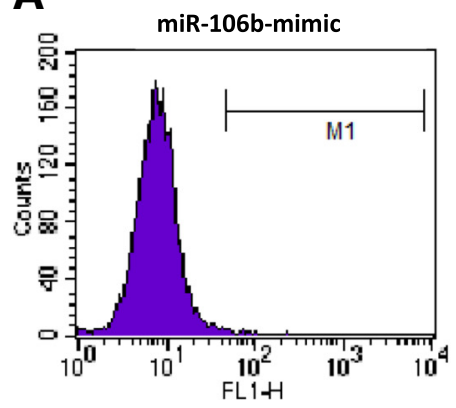

C

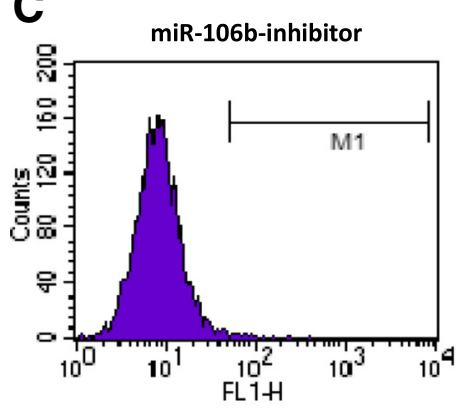

miR-106b mimic
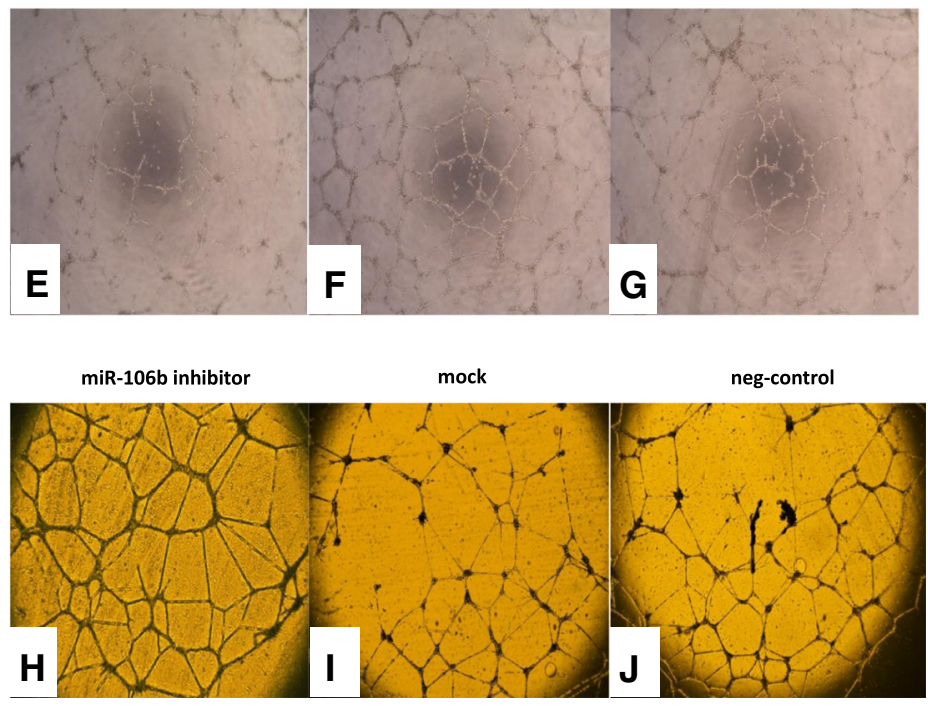

K

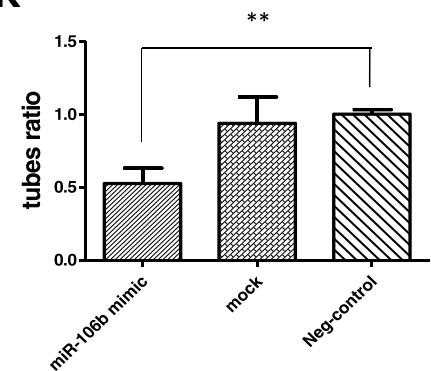

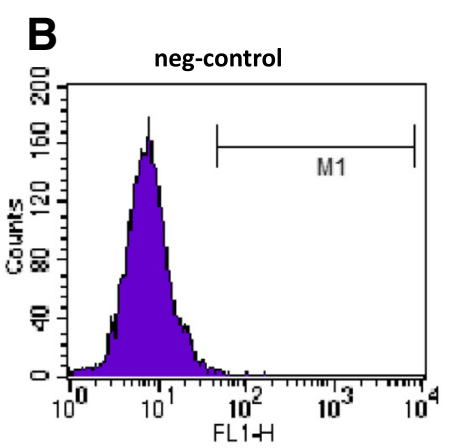

Deg-control

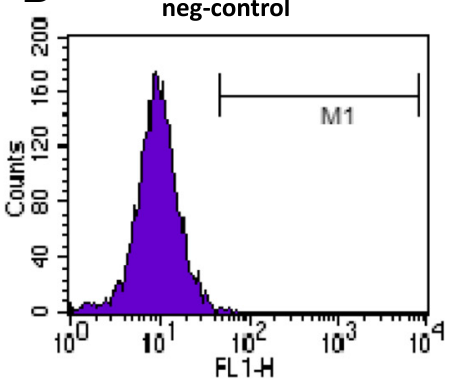

neg-control

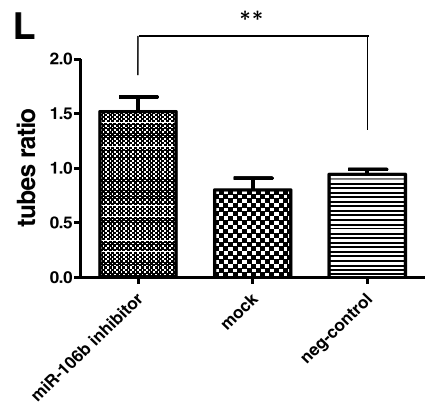

Fig. 2 (See legend on next page.) 
(See figure on previous page.)

Fig. 2 a-I Flow cytometry analysis and tube formation assay of HUVECs after transfection. a-d X axis represents fluorescence intensity, while $Y$ axis represents counts of HUVECS. M1 labeled interval stands for the FITC fluorescence intensity range identified by flow cytometry. No matter transfected with miR-106b mimic or miR-106b inhibitor, most of the HUVECs were on the left side of M1 interval, meaning that most of the cells were not apoptotic so they can't be labeled by fluorescence. e-g Less tubes were formed in the miR-106b mimic transfection group than the other two groups, furthermore, the discontinuity of tube wall was more significant than the other two groups as well (of 10x magnification). h-j On the contrary, HUVECs of miR-106b inhibitor transfection group formed more tubes with more intactness than the other two groups (of $25 \times$ magnification). $\mathbf{k}$, I Differences between different treatment groups are significant, data are presented as means \pm SEM. ${ }^{* *} P<0.05, n=4$

significantly decreased miR-106b levels (Fig. 1), which precisely confirmed that transfection procedures were successfully accomplished.

\section{Changes in general function of HUVECs after transfection} After transfection, we detected the apoptosis rate of the HUVECs by using TUNEL assay. There was no significant difference in cellular apoptosis after transfection with the different compounds (Fig. 2a-d). The overexpression of miR-106b led to less cell tube formation in the planar Matrigel assay. In contrast, the reduction of miR-106b levels by inhibitor transfection caused increased endothelial tube formation (Fig. 2e-j). Taken together, these findings reveal that miRNA-106b has anti-angiogenic properties in endothelial cells while no obvious effect on apoptosis.

\section{Screening the targets of miR-106b}

Knowing that miR-106b regulates angiogenesis in endothelial cells, we got down to finding out the potential target genes of miR-106b which could be the most key processes involved in the observed effects we mentioned above. A combination of bioinformatic algorithms (Targetscan, MiRanda and PicTar) were used to predict candidate target genes related to angiogenesis, as a result, VEGFA, JAK1, STAT3, MAPK14, IL-8(Interleukin-8), PDGFRA(Platelet-Derived Growth Factor Receptor A) were selected.
Through reviewing articles, we summarized several target genes of miR-106b $\sim 25$ cluster which were validated by other researches (Table 1 ).

In order to find out how those target genes take part in the angiogenesis processes, we acquired several key signaling pathways involving the candidate target genes by searching currently known signaling pathways related to angiogenesis through Kyoto Encyclopedia of Genes and Genomes (KEGG), the result showed VEGFA, JAK1, STAT3 and MAPK14 all have irreplaceable roles in angiogenesis (Fig. 3a).

At last, we displayed all the predicted target genes and explored the intersections among them. Then we eventually focused on VEGFA, JAK1, STAT3 and MAPK14 (Fig. 3b).

\section{MiR-106b reduces STAT3 expression in endothelial cells}

Given that miRNAs function mainly by mRNA repression, we were particularly interested in down-regulated mRNAs because they may be direct miRNA targets. Analysis performed by qRT-PCR revealed that VEGFA, $J A K 1$ and MAPK14 expression was unaffected by miR$106 \mathrm{~b}$ up or down regulation in HUVECs while STAT3 was regulated by miR-106b as revealed by qRT-PCR and also by Western blot. Our study manifested that overexpressed miR-106b represses STAT3 expression not only on mRNA but also on protein levels in endothelial cells, while miR-106b inhibition give rise to STAT3 upregulation (Fig. 4).

Table 1 Currently-known biological functions of miR-106b 25 cluster

\begin{tabular}{|c|c|c|c|}
\hline microRNAs & Biological function & Target genes & References \\
\hline miR-93 & Preventing the progression of diabetic nephropathy & VEGF & {$[22]$} \\
\hline miR-106b 25 & Inhibiting cell-cycle arrest and apoptosis & p21,Bim & {$[15,23]$} \\
\hline miR-106b & Impairing cellular cholesterol efflux and increasing the levels of secreted amyloid $\beta$ & $A B C A 1$ & {$[24]$} \\
\hline miR-106b,93 & Inhibiting brown adipocyte differentiation & & {$[25]$} \\
\hline miR-106b 25 & Inhibiting apoptosis and promoting tube formation & PTEN & {$[16]$} \\
\hline $\operatorname{miR}-25$ & Suppressing Cdk inhibitors and promoting cell proliferation & p57Kip2 & {$[23]$} \\
\hline miR-106b & Inhibiting cell cycle arrest and promoting cell proliferation & $E 2 F 1, E 2 F 3$ & {$[26]$} \\
\hline miR-106b & Activating p73 apoptotic signaling in Chronic Lymphocytic Leukemia cells & ITCH & {$[27]$} \\
\hline miR-106b & Maintaining the structural homeostasis of developing lung epithelium & MAPK14,STAT3 & {$[17]$} \\
\hline miR-93 & Inducing embryonic stem cell differentiation & STAT3 & {$[28]$} \\
\hline
\end{tabular}


A

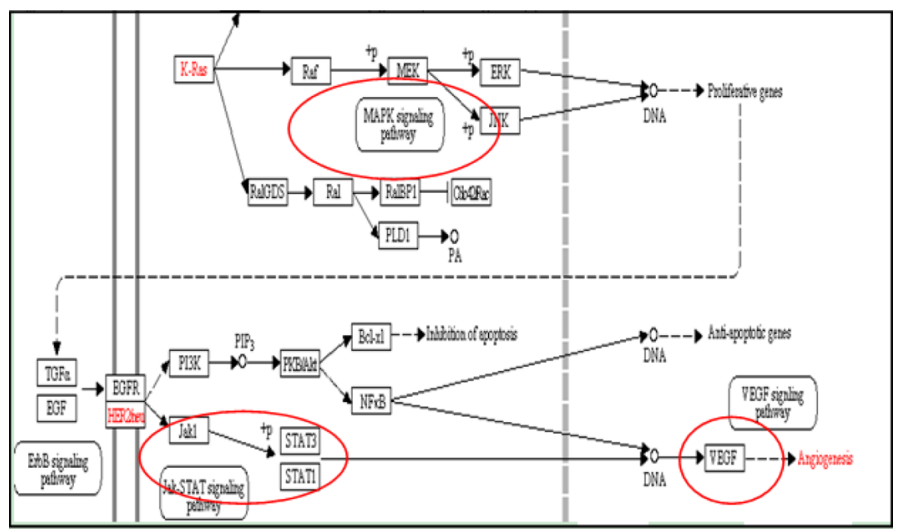

B

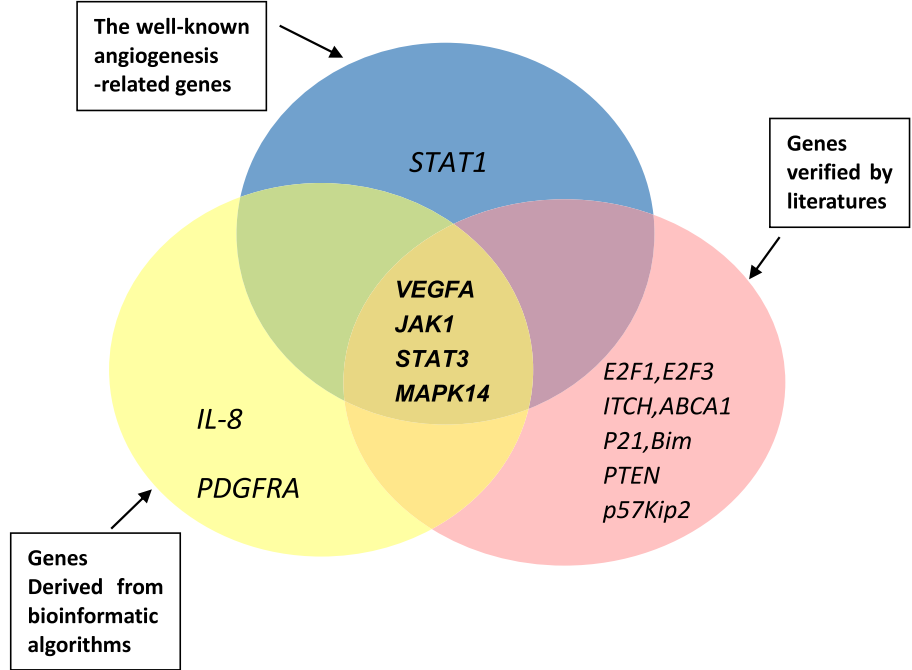

Fig. 3 a, b Signaling pathway obtained from KEGG database and schematic diagram of searching candidate target genes. a Among all the pathway, we could easily find the genes associated with angiogenesis, which are, respectively, MAPK14, JAK1, STAT1, STAT3, and VEGF. b We merged genes derived from bioinformatics algorithms, genes proven by literatures and famous angiogenesis-related genes to confirm the final candidate target genes, which are VEGFA, JAK1, STAT3 and MAPK14

\section{MiR-106b interfere STAT3's expression by direct target} impact in endothelial cells

To identify whether STAT3 is a direct target of miR106b, we constructed a luciferase reporter vector encoding the complete 3'UTR of STAT3 [WT (Wildtype) STAT3 3'UTR] as well as a control vector containing mismatches in predicted miR-106b binding site [Mut (Mutant) STAT3 3'UTR] (Fig. 5a). Co-transfection of the WT STAT3 3'UTR plasmid and miR-106b mimic in 293 T cells showed obvious decrease in fluorescence intensity whereas Mut STAT3 3'UTR didn't change luciferase gene expression, which validated that STAT3 mRNA is a direct target of miR-106b (Fig. 5b).

\section{Discussion}

Notably, miR-106b $\sim 25$ cluster, miR-17 92 cluster and miR-106a $\sim 363$ cluster are paralogs [9], which are located on different chromosomes, derived from a unique gene that underwent a series of duplications, mutations, and loss of individual miRNAs during the early evolution of vertebrates, resulting in the selection of similar but not identical clusters. Based on their seed sequences which are the regions considered most important for target selection (nucleotides 2-7), the miRNAs of these clusters can be grouped into four families: the miR-17 family (miR-17, miR-20a/b, miR-106a/b, and miR-93); the miR-18 family (miR-18a/b); the miR-19 family (miR-19a/b); and the miR-25 family (miR-25, miR-92a, and miR-363). Up till now, plenty of researches have revealed us a profile of up-regulated miR-17 92 cluster in tumors [10-12], which could promote angiogenesis [13]. Exceptionally, miR-92a has been proven to inhibit angiogenesis in vitro and in vivo [14]. Petrocca et al. [15] discovered that the miR-106b $\sim 25$ cluster could be 


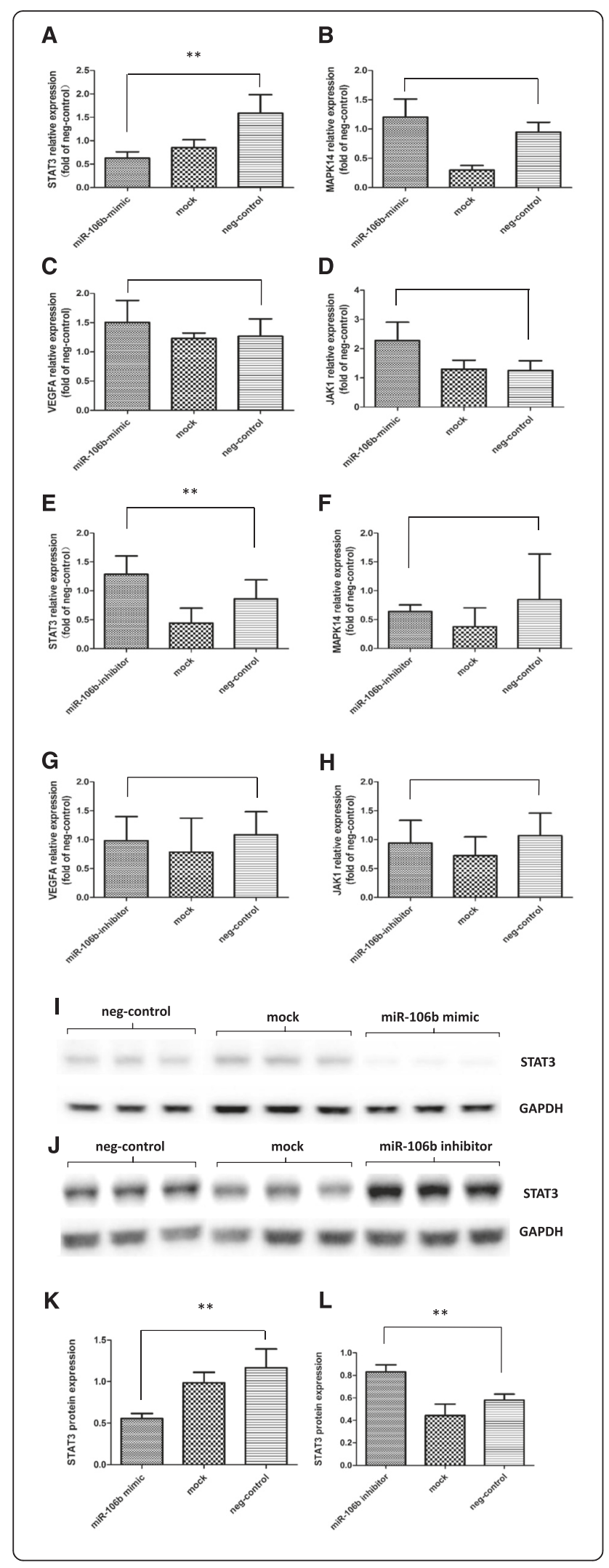

Fig. 4 a-I QRT-PCR results of different candidate genes and westernblot results of STAT3 protein expression among groups under different treatments. a-h VEGFA, JAK1 and MAPK14 expression levels are not significantly affected by miR-106b up or down regulation in HUVECs $(P>0.05, n=6)$. Meanwhile, over-expressed miR-106b represses STAT3 mRNA expression in HUVECs, while miR-106b inhibition lead to an increase in STAT3 expression. Results are shown as mean \pm SEM representative of at least three independent experiments. ${ }^{*} P<0.05$, $n=6$. $\mathrm{i}-\mathrm{I}$ Identical to the outcome obtained from qRT-PCR, miR-106b mimic transfection inhibits STAT3 expression while miR-106b inhibitor transfection enhances STAT3 expression. Data are shown as mean \pm SEM representative of at least three independent experiments. ${ }^{*} P<0.05, n=6$

activated by E2F1 in a subset of human gastric tumors. In turn, miR-106b and miR-93 regulated E2F1 expression. Eventually, up-regulation of these miRNAs impaired the TGF $\beta$ tumor suppressor pathway through interfering with the expression of CDKN1A (p21 ${ }^{\text {Waf1/Cip1 })}$ and BCL2L11 (Bim). Interestingly, miR-17 92 cluster synergistically participates in this process. As we previously stated, miR$17 \sim 92$ cluster has been proven pivotal in angiogenesis, given the similar biological functions of these two clusters, miR-106b $\sim 25$ cluster could probably be involved in angiogenesis as well. This idea was recently confirmed by Semo et al [16], who discovered that post-ischemic vascularization in miR-106b $\sim 25$ knockout mice models of hindlimb ischemia is impaired and subsequent reexpression of miR-106b $\sim 25$ via local injection of plasmids encoding this cluster can partially reverse the blood supplies. Meanwhile miR-106b $\sim 25$ knockout bone marrow stromal cells(BMSCs) shows the susceptibilities to apoptosis and decreasing abilities of paracrine and tube formation. All these suggest a pro-angiogenic effect of miR-106b 25 cluster in BMSCs, but the conclusions about miR-106b $\sim 25$ 's pro-angiogenic role in endothelial cells seems not that persuasive, since over-expression of miR-106b $\sim 25$ in H5V cells has no effect on the tube formation in vitro Matrigel assay. Therefore, to date, the specific role of miR-106b $\sim 25$ related to angiogenesis in endothelial cells is still obscure. In this study we investigated miR-106b's role in angiogenesis through observing changes in general functions of endothelial cells after up and down regulating miR-106b's expression, which demonstrates that miR-106b over-expression in endothelial cells inhibits tube formation while the reduction of miR106b's level shows the opposite. No significant difference in TUNEL assay suggests that miR-106b's expression has no obvious effect on apoptosis in endothelial cells.

Knowing that miR-106b modulates angiogenesis in endothelial cells, we wondered how these processes could be achieved. Since miRNAs function through inhibiting expression of their target genes, seeking out the potential target genes of miR-106b would explain the pivotal mechanisms giving rise to the observed effects. 


\section{A}

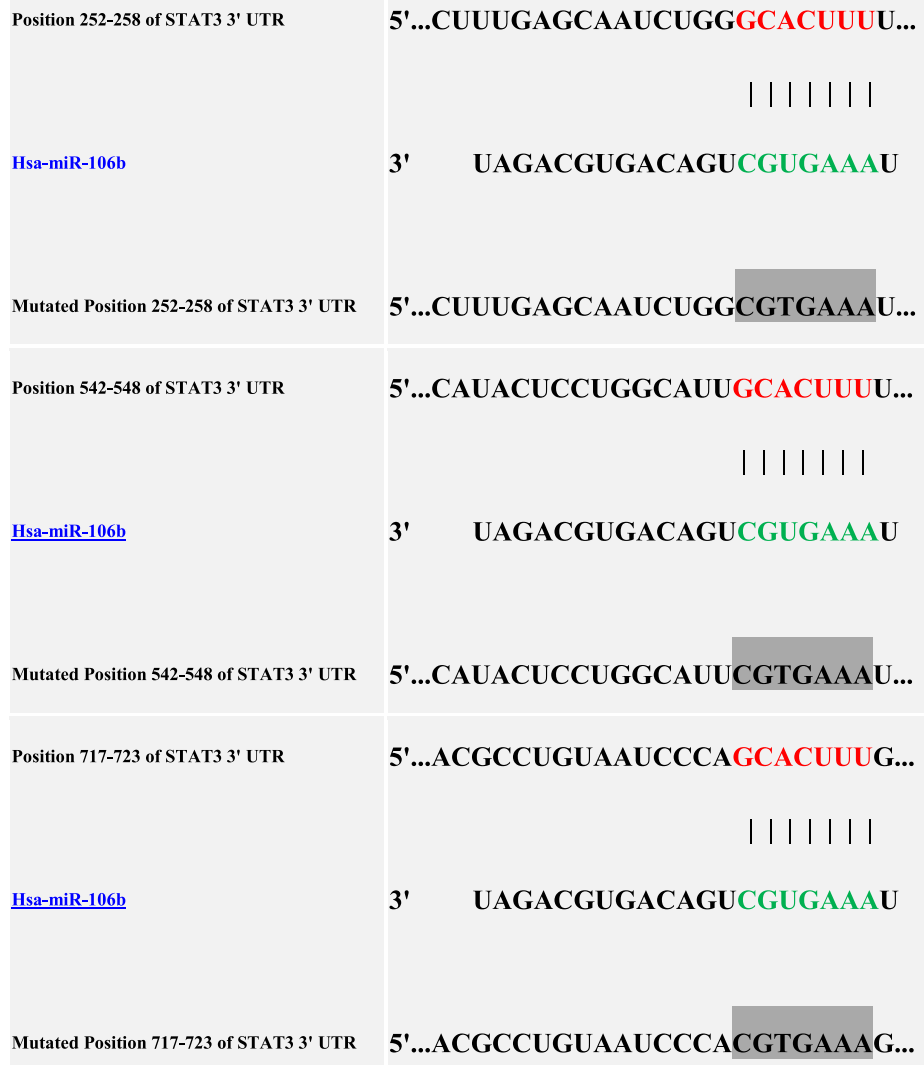

B

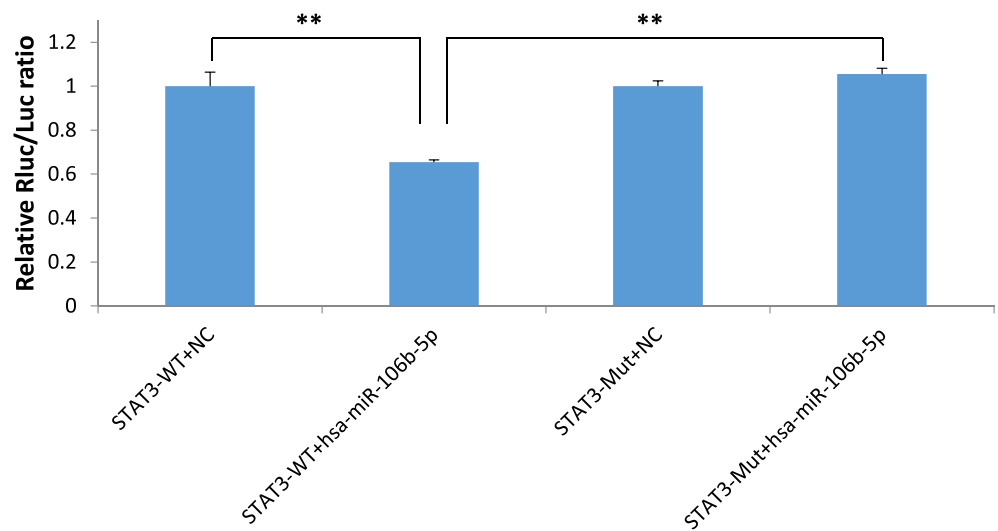

Fig. $\mathbf{5}$ a, b Luciferase assays of miR-106b binding sites on human STAT3 3'UTR. a Schematic representation of the $3^{\prime}$ untranslated region (3'UTR) of STAT3 mRNA with the predicted target sites for miR-106b. The seed sequences of miR-106b are in green letters, the target sites of STAT3 mRNA are in red letters, and the mutated miR-106b-binding sites are in gray boxes. b HUVECs were transfected with either wild-type 3'UTR STAT3 or mutant 3'UTR STAT3, along with the miR-106b mimic. hRluc (renilla luciferace gene) was reporter gene, while hluc (firefly luciferace gene) was reference gene as internal control. Results are shown as mean \pm SEM representative of three independent experiments, there are significant differences in fluorescence intensity between STAT3-WT + NC transfection group and STAT3-WT + miR-106b mimic transfection group and also between STAT3-WT + miR-106b mimic transfection group and STAT3-MUT + miR-106b mimic transfection group.** $P<0.05, n=3$

Angiogenesis-related target genes were screened out via a combination of bioinformatic algorithms (Targetscan, MiRanda and PicTar), consequently, VEGFA, JAK1, STAT3, MAPK14, IL-8, PDGFRA were selected to be the candidate genes. With the purpose of choosing the most likely genes, we consulted a certain amount of literatures to search currently known target genes associated with miR-106b $\sim 25$ cluster. As a member of miR-17 family, 
miR-106b shares an identical seed region with other family members. Therefore, it can be inferred that they may share a number of target genes. Studies showed miR-17 family contributes to maintain the structure stability of alveolar epithelial cells in growth and development process by targeting MAPK14 and STAT3 [17], meanwhile other evidence demonstrated that miR-17 family function as direct endogenous repressors of the VEGFA in nasopharyngeal carcinoma cells [18]. Researches of endothelial cells manifested that miR-17 has an anti-angiogenic function by inhibiting the expression of target gene JAK1 and subsequent repression of JAK/STAT signaling in endothelial cells [19]. Kyoto Encyclopedia of Genes and Genomes (KEGG) provided us with several angiogenesis-associated signaling pathways involving VEGFA, JAK1, STAT1, STAT3 and $M A P K 14$, which are turn out to be crucial in angiogenesis processes.

Taken together, we speculate that MAPK14, STAT3, $V E G F A$ and JAK1 could be the potential target genes of miR-106b. Based on this speculation, our study focused on how miR-106b act on its target in endothelial cell-related angiogenesis. Data gathered from the qRT-PCR and Western blot demonstrated that up or down regulation of miR-106b in HUVECs has no influence on expression of VEGFA, JAK1 and MAPK14, while expression of STAT3 would be repressed by miR-106b. Therefore, there is every reason to suspect that miR-106b inhibits STAT3 expression via directly binding to it. Consistent with Carraro $G$ et al [17], luciferase reporter assay showed obvious decrease in fluorescence intensity when WT STAT3 3'UTR plasmid and miR-106b mimic co-transfected together whereas Mut STAT3 3'UTR didn't change luciferase gene expression, which verified that miR-106b inhibits STAT3 expression by a direct target effect. Thus, our research explicitly revealed miR-106b suppresses STAT3 expression, which due to a direct effect mediated by miR-106b, that is to say, miR-106b exhibits an anti-angiogenic function by inhibiting STAT3 expression in endothelial cells.

In this research, we have confirmed miR-106b directly inhibited STAT3 expression with no obvious effect on $M A P K 14, V E G F A$ and $J A K 1$, which was partially consistent with Carraro's [17] discovery that miR-17 family downregulate STAT3 and MAPK14 expression in lung epithelial cells. As is known to all, STAT3 pathway was identified as key components linking cytokine signals to transcriptional events in cells, meanwhile STAT proteins can regulate many pathways including cell-cycle progression, apoptosis, tumor angiogenesis, tumor-cell invasion and metastasis, and tumor-cell evasion of the immune system [20]. Niu et al [21] demonstrated that VEGF expression correlated with STAT3 activity in various human cancer cell lines. An activated STAT3 mutant upregulated VEGF expression and promoted tumor angiogenesis. Moreover, interrupting STAT3 signaling in tumor cells downregulated VEGF expression and inhibited angiogenesis. Apparently, STAT3 pathway promote angiogenesis primarily through upregulating VEGFA expression, however, our results showed that VEGFA level was not affected. We considered STAT3 may regulate other angiogenic factors, such as HIF- $1 \alpha$ and FGF2, to promote angiogenesis process. Originally, our research was designed to find out miR-106b's function in endothelial cells based on the predicted target genes of miR106b through TargetScan/MiRanda/PicTar algorithms, according to which, HIF-1 $\alpha$ and FGF2 are not predicted target genes of miR-106b. Therefore, detecting those factors does not conform to our original research intention. Nevertheless, in our subsequent research plan, we are focusing on this issue to ensure its completion.

\section{Conclusions}

Our research found that miR-106b restrains angiogenesis processes in endothelial cells, meanwhile expression of STAT3 is significantly repressed, which was subsequently proven to be a direct effect between miR-106b and STAT3. Given the importance of STAT3 in angiogenesis, we conclude that miR-106b exhibits an anti-angiogenic effect in endothelial cells through directly binding to STAT3.

\section{Ethics approval and consent to participate \\ Not applicable.}

\section{Consent for publication}

Not applicable.

\section{Availability of data and materials}

The raw data cannot be shared for now, since the data may be used in our subsequent researches.

\section{Abbreviations \\ GAPDH: Glyceraldehyde-3-phosphate dehydrogenase; IL-8: Interleukin-8; JAK1: Janus kinase 1; KEGG: Kyoto encyclopedia of genes and genomes; MAPK14: Mitogen-activated protein kinase; Mut: Mutant; PDGFRA: Platelet-derived growth factor receptor A; PVDF: Polyvinylidene fluoride; RIPA: Radio-immunoprecipitation assay; SDS-PAGE: Sodium dodecyl sulfate polyacrylamide gel electrophoresis; STAT3: Signal transducers and activators of transcription 3; VEGFA: Vascular endothelial growth factor A; WT: Wildtype. \\ Competing interests \\ The authors declare that they have no competing interests. \\ Authors' contributions \\ YM designed research and critically revised the manuscript. AM designed research, performed research, analyzed data and wrote the manuscript. AM performed research and analyzed data. YY critically revised the manuscript. All authors read and approved the final manuscript.}

\section{Authors' information}

Ailifeire Maimaiti M.D. graduated from Peking University Health Science Center now works in the First Affiliated Hospital of Xinjiang Medical 
University as an attending physician. During her M.D. study, her research focused mainly on microRNAs and atherosclerosis. In this study, they demonstrated that STAT3 is a direct target of miR-106b which functions as an angiogenesis inhibitor. Aikebaier Maimaiti who also works as an attending physician dedicated a lot by performing research and analyzing data. Yitong Ma and Yining Yang are experienced chief physicians and professors in cardiology department who have cultivated a galaxy of great physicians in Xinjiang and also rendered many valuable advice on this research.

\section{Acknowledgements}

This research was also supported by Guangzhou RiboBio.Co.Ltd.

\section{Funding}

This research was supported by the Innovation Team Development Program Foundation from the Ministry of Education of China(Grant No. IRT13094) and the Foundation of the First Affiliated Hospital of Xinjiang Medical University(Grant No. 2014ZRQN01).

Received: 18 December 2015 Accepted: 1 March 2016

Published online: 09 March 2016

\section{References}

1. Cao Y, Hong A, Schulten H, Post MJ. Update on therapeutic neovascularization. Cardio Res. 2005;65:639-48.

2. Carmeliet P. Angiogenesis in health and disease. Nat Med. 2003;9:653-60.

3. Hanahan D. Signaling vascular morphogenesis and maintenance. Science. 1997;277:48-50

4. Virmani R, Kolodgie FD, Burke AP, et al. Atherosclerotic plaque progression and vulnerability to rupture. Arterioscler Thromb Vasc Biol. 2005;25:2054-61.

5. O'Brien ER, Garvin MR, Dev R, et al. Angiogenesis in human coronary atherosclerotic plaques. Am J Pathol. 1994;145:883-94.

6. Moulton KS, Heller E, Konerding MA, Flynn E, Palinski W, Folkman J. Angiogenesis inhibitors endostatin or TNP-470 reduce intimal neovascularization and plaque growth in apolipoprotein E-deficient mice. Circulation. 1999;99:1726-32.

7. Fichtlscherer $\mathrm{S}$, De Rosa S, Fox H, et al. Circulating microRNAs in patients with coronary artery disease. Circ Res. 2010;107:677-84.

8. Cordes KR, Srivastava D. MicroRNA regulation of cardiovascular development. Circ Res. 2009;104:724-32.

9. Petrocca F, Vecchione A, Croce CM. Emerging role miR-106b-25/miR-17-92 clusters in the control of transforming growth factor beta signaling. Cancer Res. 2008;68:8191-4.

10. Ota A, Tagawa H, Karnan S, et al. Identification and characterization of a novel gene, C13orf25, as a target for 13q31-q32 amplification in malignant lymphoma. Cancer Res. 2004;64:3087-95.

11. He L, Thomson JM, Hemann MT, et al. A microRNA polycistron as a potential human oncogene. Nature. 2005;435:828-33.

12. Venturini $L$, Battmer $K$, Castoldi $M$, et al. Expression of the miR-17-92 polycistron in chronic myeloid leukemia (CML) CD34 ${ }^{+}$cells. Blood. 2007; 109(10):4399-405.

13. Dews M, Homayouni $A, Y u$ D, et al. Augmentation of tumor angiogenesis by a Myc-activated microRNA cluster. Nat Genet. 2006;38:1060-5.

14. Bonauer A, Carmona G, Iwasaki M, et al. MicroRNA-92a controls angiogenesis and functional recovery of ischemic tissues in mice. Science. 2009:324(5935):1710-3

15. Petrocca F, Visone R, Onelli MR, et al. E2F1-regulated microRNAs impair TGF $\beta$-dependent cell-cycle arrest and apoptosis in gastric cancer. Cancer Cell. 2008;13(3):272-86

16. Semo J, Sharir R, Afek A, et al. The 106b 25 microRNA cluster is essential for neovascularization after hindlimb ischaemia in mice. Eur Heart J. 2014; 35(45):3212-223.

17. Carraro G, El-Hashash A, Guidolin D, et al. MiR-17 family of microRNAs controls FGF10-mediated embryonic lung epithelial branching morphogenesis through MAPK14 and STAT3 regulation of E-Cadherin distribution. Dev Biol. 2009;333(2):238-50.

18. Ye W, LV Q, Wong CK, et al. The effect of central loops in miRNA: MRE duplexes on the efficiency of miRNA-mediated gene regulation. PLoS One. 2008:3(3):e1719.

19. Doebele C, Bonauer A, Fischer A, et al. Members of the microRNA-17-92 cluster exhibit a cell-intrinsic, antiangiogenic function in endothelial cells. Blood. 2010;115(23):4944-50.
20. Haura EB, Turkson J, Jove R, et al. Mechanisms of disease: insights into the emerging role of signal transducers and activators of transcription in cancer. Nat Clin Pract Oncol. 2005;2(6):315-24.

21. Niu G, Wright KL, Huang M, et al. Constitutive Stat3 activity up-regulates VEGF expression and tumor angiogenesis. Oncogene. 2002;21(13):2000-8.

22. Long J, Wang Y, Wang W, Chang BHJ, Danesh FR. Identification of microRNA-93 as a novel regulator of vascular endothelial growth factor in hyperglycemic conditions. J Biol Chem. 2010;285(30):23457-465.

23. Kim YK, Yu J, Han TS, et al. Functional links between clustered microRNAs: suppression of cell-cycle inhibitors by microRNA clusters in gastric cancer. Nucleic Acids Res. 2009;37(5):1672-681.

24. Kim J, Yoon H, Ramírez CM, et al. miR-106b impairs cholesterol efflux and increases $A \beta$ levels by repressing ABCA1 expression. Exp Neurol. 2012; 235(2):476-83

25. Wu Y, Zuo J, Zhang Y, et al. Identification of miR-106b-93 as a negative regulator of brown adipocyte differentiation. Biochem Bioph Res Co. 2013; 438(4):575-80

26. Trompeter $\mathrm{HI}$, Abbad H, Iwaniuk KM, et al. MicroRNAs miR-17, miR-20a, and miR-106b act in concert to modulate E2F activity on cell cycle arrest during neuronal lineage differentiation of USSC. PLoS One. 2011;6(1):e16138.

27. Sampath D, Calin GA, Puduvalli VK, et al. Specific activation of microRNA106b enables the p73 apoptotic response in chronic lymphocytic leukemia by targeting the ubiquitin ligase Itch for degradation. Blood. 2009; 113(16):3744-753.

28. Foshay KM, Gallicano Gl. miR-17 family miRNAs are expressed during early mammalian development and regulate stem cell differentiation. Dev Biol. 2009;326(2):431-43.

\section{Submit your next manuscript to BioMed Central and we will help you at every step:}

- We accept pre-submission inquiries

- Our selector tool helps you to find the most relevant journal

- We provide round the clock customer support

- Convenient online submission

- Thorough peer review

- Inclusion in PubMed and all major indexing services

- Maximum visibility for your research

Submit your manuscript at www.biomedcentral.com/submit 